\title{
VARIACIÓN DE LA ACTIVIDAD ANTIOXIDANTE POR EFECTO DEL TOSTADO EN GRANOS DE CAFÉ (COFFEA ARABICA), ESTADO MIRANDA, VENEZUELA
}

\section{Variation of the antioxidant activity by effect of roasting in coffee beans (Coffea arabica), Miranda state, Venezuela}

\section{Franklin Pacheco-Coello \\ Laboratorio de Metales Pesados-Solventes Orgánicos \\ y Sección de Biotecnología Agroindustrial del Insti- tuto de Investigaciones Biomédicas "Dr. Francisco J. Triana Alonso" (BIOMED-UC). Aragua, Venezuela. ORCID: 0000-0002-2765-4069 \\ Correo-e: pachecofranklin74@gmail.com \\ Roxana Torres \\ Laboratorio de Metales Pesados-Solventes Orgánicos Aragua, Venezuela. ORCID: 0000-0003-3806-3122 Correo-e: vanes7781@gmail.com}

\author{
Teiron Arvelo \\ Laboratorio de Metales Pesados-Solventes Orgánicos. \\ Aragua, Venezuela. ORCID: 0000-0001-7053-716X \\ Correo-e:pascalsuloaga@gmail.com

\section{Ismar Velásquez} \\ Laboratorio de Metales Pesados-Solventes Orgánicos. \\ Aragua, Venezuela. ORCID: 0000-0002-6904-4613 \\ Correo-e: yulyfer02@gmail.com
}

Recibido: 23-X-2020 • Aprobado: 26-XI-2020

Cómo citar: Pacheco-Coello, F., Torres, R., Arvelo, T., \& Velasquez, I. (2020). Variación de la actividad antioxidante por efecto del tostado en granos de café (Coffea arabica), estado Miranda, Venezuela. Ciencia, Ambiente y Clima, 3(2), 49-56. Doi: https://doi. org/10.22206/cac.2020.v3i2.pp49-56

\section{Resumen}

Los compuestos fenólicos son metabolitos secundarios presentes en una diversidad de material vegetal cuya concentración, en el caso del café se ve afectada durante el proceso de tostado. La investigación tuvo como objetivo determinar el efecto que tiene la temperatura de tostado en la contracción de los compuestos fenólicos totales y actividad antioxidante del café. Se contó con 300 gramos de café verde del cual 150 gramos sufrieron un proceso de tostado medio ( $195{ }^{\circ} \mathrm{C}$ aproximadamente). Se emplearon tres métodos colorimétricos para determinación de fenoles totales, flavonoides. La actividad antioxidante se evaluó por el método del radical libre 2,2-difenil-1-picrilhidrazil (DPPH) y poder reductor férrico (FRAP). Se observó una mayor concentración de fenoles totales y flavonoides en el café verde con diferencia significativa respecto al tostado $(\mathrm{p}<0,263)$. En relación a las antocianinas la contracción fue superior en el café tostado $(\mathrm{p}<0,324)$. Se observó una actividad antioxidante por el método de DPPH fue de $92,2 \%\left(\mathrm{IC}_{50}=298,4\right.$ para el extracto de café verde y de $79,3 \%$ para café tostado. Del estudio se concluye que la

\begin{abstract}
Phenolic compounds are secondary metabolites present in a variety of plant material whose concentration, in the case of coffee, is affected during the roasting process. The research aimed to determine the effect that the roasting temperature has on the contraction of total phenolic compounds and antioxidant activity of coffee. There were 300 grams of green coffee of which 150 grams suffered an average roasting process $\left(195^{\circ} \mathrm{C}\right.$ approximately). Three colorimetric methods were used for the determination of total phenols, flavonoids. The antioxidant activity was evaluated by the method of the free radical 2,2-diphenyl-1-picrylhydrazil (DPPH) and ferric reducing power (FRAP). A higher concentration of total phenols and flavonoids was observed in green coffee with significant difference from roasting $(\mathrm{p}<0.263)$. In relation to anthocyanins the contraction was higher in roasted coffee $(\mathrm{p}<0.324)$. The antioxidant activity by DPPH method was $92.2 \%\left(\mathrm{IC}_{50}=298.4\right.$ for green coffee extract and 79.3 $\%$ for roasted coffee. From the study it is concluded that temperature has an impact on the contraction of phenolic
\end{abstract}


temperatura repercute en la contracción de los compuestos fenólicos presentes en el café, influyendo en la actividad antioxidante de una de las bebidas más consumidas a nivel mundial.

Palabras clave: fenoles, flavonoides, FRAP, DPPH, antioxidante

\section{Introducción}

El café (Coffea arabica) es un arbusto de la familia de las rubiáceas nativo de Etiopía, el cual alcanza los 12 metros de altura en estado silvestre, con hojas encontradas, ovales u oblongas de color verde oscuro (Miranda et al., 2007; Chávez et al., 2012) (Figura 1). El grano de café ha sido históricamente, uno de los productos más comercializado y consumido en el mundo, por su sabor y aroma característico, con casi mil compuestos volátiles (Budryn

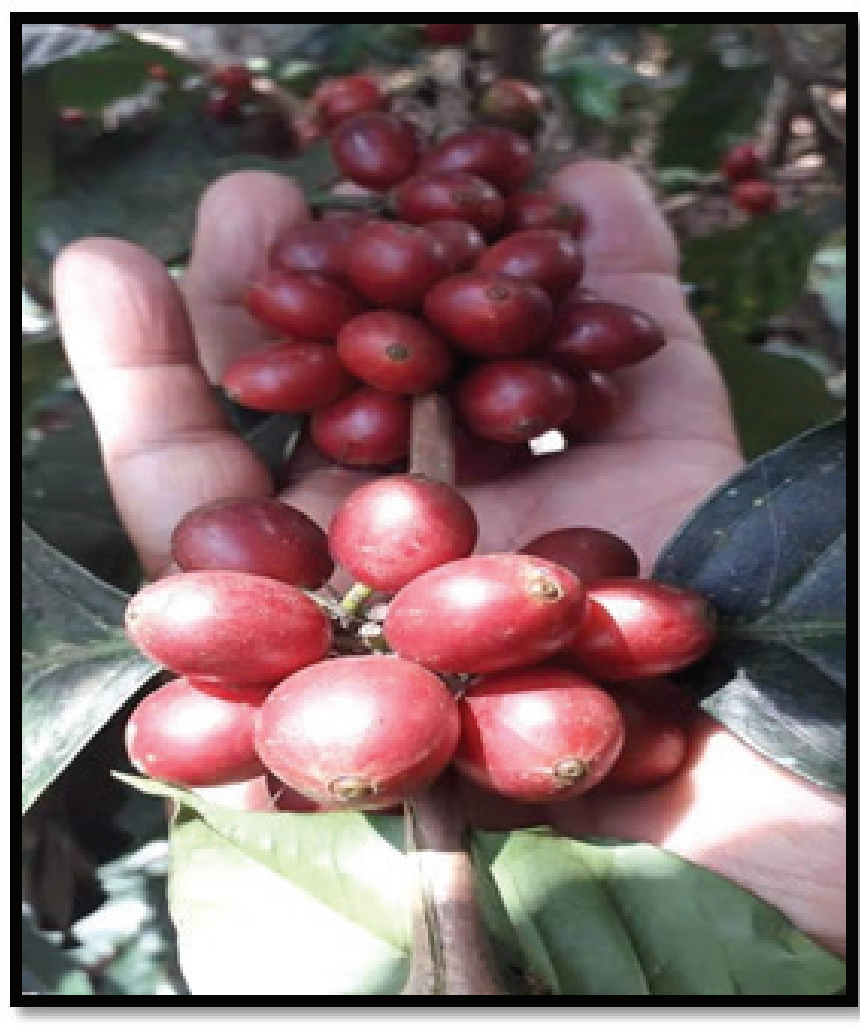

compounds present in coffee, influencing the antioxidant activity of one of the most consumed beverages worldwide.

Keywords: phenols, flavonoids, FRAP, DPPH, antioxidant.

et al., 2009; Ludwig et al., 2012; Aguiar et al., 2016). A nivel mundial es consumido como bebida caliente, catalogada como alimento funcional con capacidad antioxidante, atribuyéndosele de acuerdo a diversos estudios epidemiológicos, de prevenir diabetes tipo 2, cirrosis hepática, cáncer de hígado y colonrrectal, enfermedades cardiovasculares, inflamatorias y neurodegenerativas como el Alzheimer y Parkinson (Kocadağlıa \& Gökmen, 2016; Shang et al., 2017).

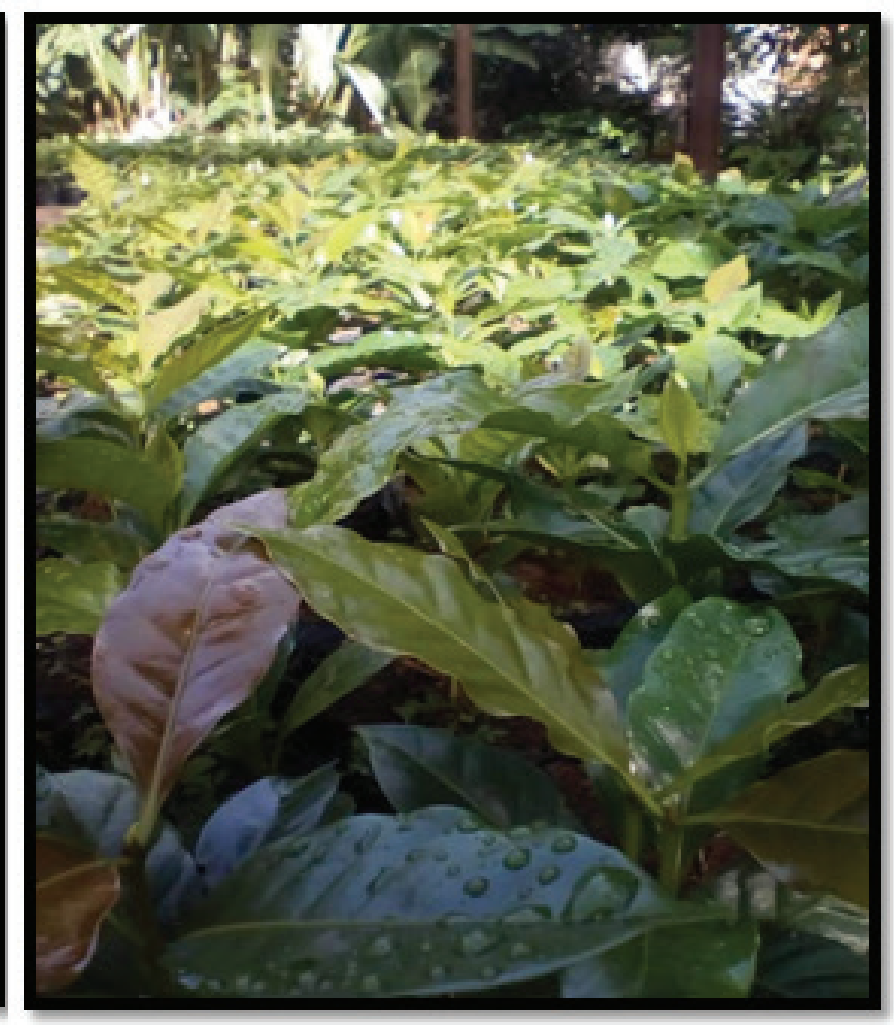

Figura 1. Fruto y planta de Coffea arabica. Fuente: Cortesía de empresa cafetera, los Teques estado Miranda 
Por otra parte, dentro de su composición química, el grano de café posee una variedad de compuestos fenólicos; tales como, flavonoides, ácidos clorogénicos, cafeico, fenílico y cumárico; que se ven afectado por la temperatura en el proceso de tostado (bajo, medio y alto), la cual dependen de cada empresa comercializadora y de la calidad del grano de café (Ribeiro et al., 2010; García et al., 2016). Este proceso de tostado es una etapa obligatoria y de gran importancia; que proporciona el producto de consumo propiamente dicho que es el café, el cual consiste en pasar los granos de café por una fuente de calor para así tomar el color y el aroma característico con que se le conoce (Schenker $e t$ al., 2002).

En tal sentido partiendo variabilidad post tostado, de los compuestos antioxidantes presentes en el café y su beneficio en la salud humana, el estudio tuvo como objetivo determinar el efecto final de la temperatura en la concentración de compuestos fenólicos totales y la actividad antioxidante de extractos de café verde y tostado (Coffea arabica), cultivados durante el año 2019 en el estado Miranda, Venezuela.

\section{Materiales y Métodos}

\section{Material vegetal}

Se contó con 300 gramos de café verde por saco muestreado ( 3 en total), utilizándose un calador de granos según la Comisión Venezolana de Normas Industriales (COVENIN 383:1995) proporcionados por la empresa, ubicada en la ciudad de los Teques, estado Miranda, Venezuela. Este fue dividido para su procesamiento y cuantificación de fenoles totales, flavonoides y evaluación de la actividad antioxidante de acuerdo al siguiente protocolo propuesto por Reyes-Luengas et al., 2012, con modificación en el contenido de la muestra:

a) 150 gramos de café verde: subdivididos en tres porciones de 50 gramos, pulverizados con un molino manual. b) 150 gramos de café tostado: subdivididos en tres porciones de 50 gramos. El proceso de tostado fue realizado por la empresa donadora de los granos de café. El tipo de tostión fue tostado medio (aproximadamente $195^{\circ} \mathrm{C}$ ). $\mathrm{Al}$ igual que el café verde, este fue pulverizado con un molino manual. Es importante destacar que por política de la empresa solo se proporcionó una temperatura aproximada. Figura 2.
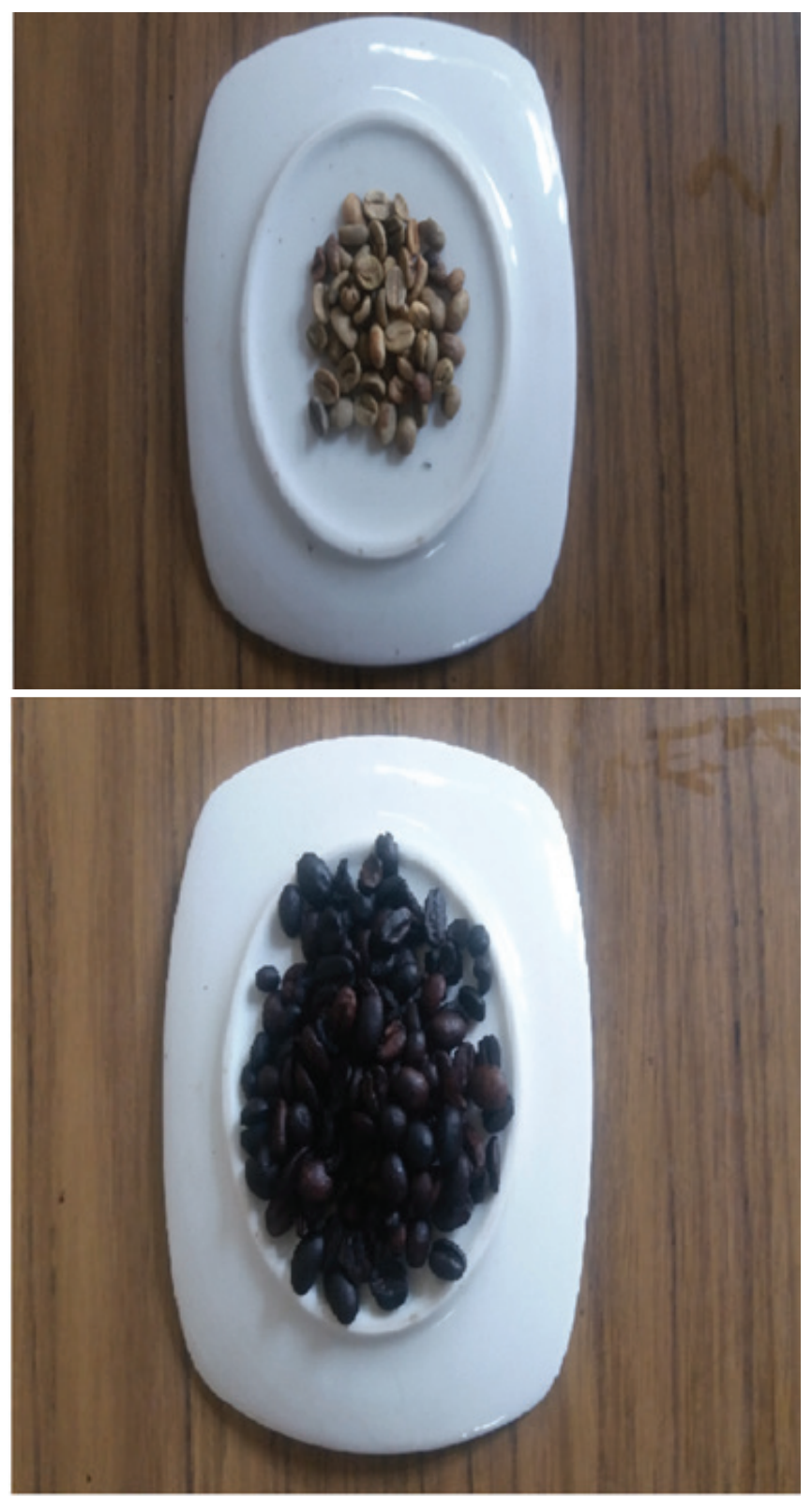

Figura 2. Granos enteros de café verde y tostado. Fuente: Elaboración propia 


\section{Obtención del extracto acuoso}

Para la obtención del extracto acuoso se mezcló $5 \mathrm{~g}$ de muestra (café verde molido y café tostado) con $100 \mathrm{~mL}$ de agua destilada y se colocaron en una plancha de calentamiento y mantuvo durante $5 \mathrm{~min}$ en ebullición. Luego se procedió a filtrar la muestra a través de papel filtro Whatman No. 1 y se aforó a 200 $\mathrm{mL}$ con agua destilada (Reyes-Luengas et al., 2012). Este procedimiento se realizó por triplicado.

\section{Determinación de fenoles totales}

Para la determinación de fenoles totales se empleó el método descrito por Jeszka-Skowron et al., 2015. Las lecturas de absorbancias se midieron en un espectrofotómetro UV/VIS Génesis 20 (Thermo Scintific, Waltham, Massachusetts, USA). Se preparó una curva de calibración con un estándar de Ácido Gálico (Sigma-Aldrich, Berlín Alemania) a concentraciones de 50, 100, 200, 300, 400, 500 y 1000 ppm. Los resultados fueron expresados en mg equivalentes de ácido gálico por gramo de café molido (mg EAG/ g CM).

\section{Determinación de flavonoides}

Para la determinación de flavonoides se empleó el método descrito por Marinova et al., 2005. $100 \mu \mathrm{L}$ de extracto fueron mezclados con $30 \mu \mathrm{L}$ de $\mathrm{NaNO}_{2}$ al 5\% (p/v), $30 \mu \mathrm{L}$ de $\mathrm{AlCl}_{3} 10 \%(\mathrm{p} / \mathrm{v}), 200 \mu \mathrm{L}$ de $\mathrm{NaOH}$ a $1 \mathrm{M}$ y completándose con agua destilada hasta un volumen final de $1000 \mu \mathrm{L}$. Las lecturas espectrofotométricas se realizaron a $510 \mathrm{~nm}$ y se comparó con la curva patrón usando como estándar (+)-catequina $(50,100,150,200$ y $250 \mu \mathrm{g} / \mathrm{mL})$. Los resultados fueron expresados como mg Equi- valente de Catequina por gramos de café molido (mg EC / g CM).

\section{Actividad Antioxidante}

\section{Método del radical libre 2,2-difenil-1-picrilhi- drazil (DPPH).}

Para evaluar la actividad antioxidante se usó el método del radical libre 2,2-difenil-1-picrilhidrazil (DPPH) (Sigma Aldrich $\mathrm{Co}^{\circ}$ ) con una solución 100 $\mu \mathrm{M}$ de DPPH en metanol al $80 \%$, la cual permite observar una disminución de la absorbancia, debido a la cesión de un átomo de hidrogeno por parte de los compuestos antioxidantes presentes en los extractos. En una cubeta de vidrio se colocaron 100 $\mu \mathrm{L}$ de extracto y $2,9 \mathrm{~mL}$ de DPPH. La absorbancia se monitoreó cada 5 min por 30 min a una longitud de onda de $515 \mathrm{~nm}$. La absorbancia de referencia $\left(A_{0}\right)$ fue obtenida al sustituir el volumen de extracto por metanol al $80 \%$. El porcentaje de reducción de DPPH se obtuvo de la expresión: DPPH (\%)= $\left(A_{0}-A_{n}\right) 100 / A_{0}$, donde $A_{0}$ y $A_{n}$ fueron las absorbancias de referencia y de la muestra, respectivamente (Soler-Rivas et al., 2000).

\section{Determinación de la capacidad antioxidante por el ensayo del poder reductor férrico (FRAP).}

Este ensayo permite determinar la capacidad reductora de los extractos (Benzie \& Strain, 1996). Para esto $100 \mu \mathrm{L}$ de cada extracto se mezcló con $3 \mathrm{~mL}$ de reactivo FRAP (300 mM de tampón de acetato de sodio y ácido acético, solución de diamonio de 2, 4,6-tri (2-piridil)-s-triazina) de $10 \mathrm{mM}$ y solución $\mathrm{FeCl}_{3} 20 \mathrm{mM}$ ), en una relación de volumen 
de 10: 1: 1. Se dejó reposar a temperatura ambiente durante 4 minutos, para luego leer las absorbancias a $593 \mathrm{~nm}$, empleando un espectrofotómetro UV/ VIS Génesis 20 (Thermo Scintific,). Se empleó $\mathrm{FeSO}_{4}$ como estándar y los resultados se expresaron en $\mu \mathrm{mol} \mathrm{Fe}{ }^{+2} / \mathrm{g} \mathrm{CM}$.

\section{Análisis estadístico.}

Todas las determinaciones se realizaron por triplicados y se expresaron los valores como los promedios \pm la desviación estándar (DE). Para la comparación de la concentración tanto de fenoles totales, flavonoides y actividad antioxidante se aplicó un análisis de varianza de dos vías con interacción (ANOVA) pruebas de comparación de medias (Tukey; $p \leq 0,05$ ) usando el programa Statistix 9.0 para Windows.

\section{Resultados y Discusión}

Un aspecto fundamental para obtener un café de alta calidad y que conserve el mayor contenido de sustancias químicas que aporten beneficios al consumidor, es el tostado, etapa unitaria más importante en la tecnología del café, en la cual se fijan y forman químicamente sustancias orgánicas que originan el aroma, color, olor y sabor que se verán reflejados en la taza (Garay, 2014). El análisis de los extractos arrojó que la concentración de fenoles totales y flavonoides resultó ser mayor en café verde, con una notable disminución de dichos compuestos post tostado, observándose además una significancia estadística al comparar ambos extractos $(p<0,05)$.

Tabla 1. Concentración de fenoles totales (mg EAG/g café) y flavonoides (mg CE/g café).

\begin{tabular}{|c|c|c|c|}
\hline Material vegetal & $\begin{array}{c}\text { Café verde } \\
\text { Media } \pm \text { DS }\end{array}$ & $\begin{array}{c}\text { Café tostado } \\
\text { Media } \pm \text { DS }\end{array}$ & $\boldsymbol{p}$ \\
\hline Fenoles Totales & $43,42 \pm 0,12$ & $30,03 \pm 0,91$ & 0,027 \\
\hline Flavonoides & $32,01 \pm 0,49$ & $19,89 \pm 0,18$ & 0,031 \\
\hline
\end{tabular}

Nota: $\left(^{*}\right)$ Significativo si $p<0,05$. DS= desviación estándar.

Fuente: Base de datos investigación 2019. 
Estos resultados son comparables con la hallado por Odžakovi et al., 2016, el cual observó que la concentración de fenoles totales y flavonoides disminuyó posterior al proceso de tostado. Así mismo Król et al., 2020, reportaron una diferencia estadística al comparar la concentración de compuestos fenólicos totales en granos de café verde y sometidos a tostado ligero, medio y alto.

Por otra parte, diversos estudios indican que, para evaluar la actividad antioxidante de cualquier tipo de extracto natural, es indispensable el empleo de dos o más métodos (Fu et al., 2011; Gan et al., 2017). En este sentido con el método FRAP se observó un mayor poder reductor en el extracto de café verde $(149,34 \pm 1,48 \mu \mathrm{mol} \mathrm{Fe}+2 / \mathrm{g} \mathrm{CM})$ en comparación con el tostado $(99,12 \pm 1,09 \mu \mathrm{mol}$ $\left.\mathrm{Fe}^{+2} / \mathrm{g} \mathrm{CM}\right)$; arrojando además diferencia estadística $(\mathrm{p}=0,025)$. En relación al método DPPH, el porcentaje de reducción y el $\mathrm{IC}_{50}$, que representa la concentración a la cual se obtiene el $50 \%$ de reducción del DPPH se observan en la Figura 3.

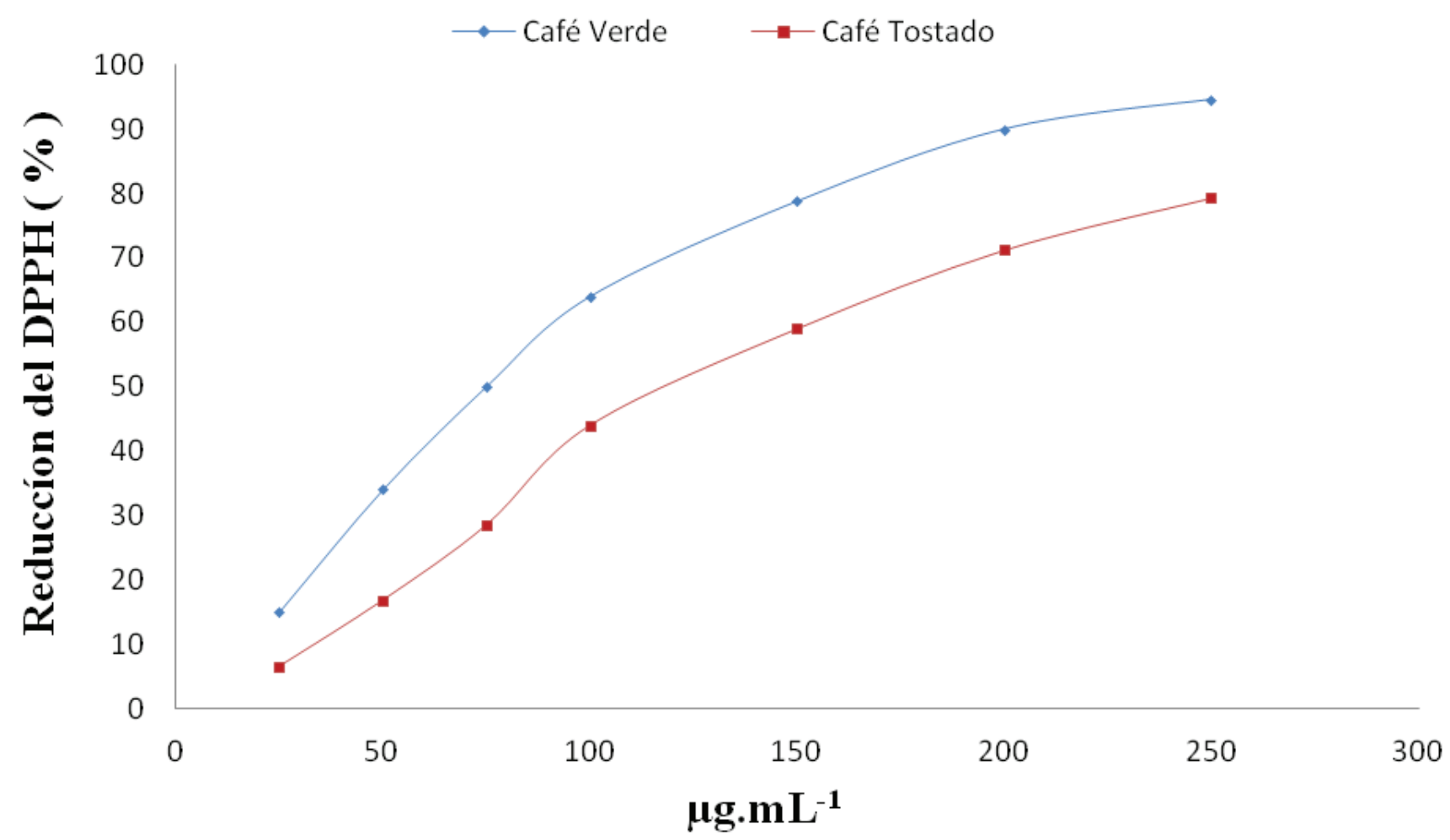

Figura 3. Comparación del a antioxidante de los extractos de café verde y tostado por método DPPH.

Fuente: Elaboración propia

La diferencia observada puede deberse a que el café verde posee compuestos fenólicos, como los ácidos clorogénicos, cafeicos, fenílico y cumárico, que al tostarse afecta marcadamente la composición de fenoles debido a la reacción de Maillard (Gutiérrez, 2002). Estos resultados indican que la actividad antioxidante del café depende de una variedad de componentes bioactivos que se ven alterados en cuanto a su concentración una vez que estos son tostados (Jeszka-Skowron et al., 20 15; Odžakovi et al., 2016; Benigno et al., 2018; Lazcano et al., 2015). Por último vale la pena resaltar que la presencia de estos compuestos en el grano de café verde, también depende de la variedad y origen del

54 | Ciencia, Ambiente y Clima 2020; 3(2, julio-diciembre): 49-56 
grano, condiciones ambientales propias de cada región, tipo de suelo, genética de la planta entre otras (Ramírez et al., 2011).

\section{Conclusiones}

En el presente estudio se evidenció que el proceso tostado influye en la concentración de los compuestos fenólicos, que ha su vez afecta directamente la capacidad antioxidante de este material vegetal. Cada empresa posee y se justa a normativas establecidas en cada país. Sin embargo, uno de los datos poco conocidos y que no se expresa en el empaque comercial es la temperatura de tostado, siendo categorizadas simplemente como tostado ligero o suave, medio y alto u oscuro. Si bien el estudio se limitó al efecto del tostado, vale resaltar que la calidad del grano de café juega un papel importante en la expresión de los compuestos fenólicos.

\section{Referencias}

Aguiar, J., B. N. Estevinho, B. N. \& Santos, L. (2016). Microencapsulation of natural antioxidants for food application - The specific case of coffee antioxidants, Trends in Food Science \& Technology, 58, 21-39.

Benigno, M. E., Fong, L. E., Biju, D., Muharram, A., Davis, I. M., Vela, K. O., Rios D, Osorio-Camacena E, Kaur B., Rojas S. \& Forester, S. C. (2018) The impact of the roast levels of coffee extracts on their potential anticancer activities. Journal Food Science, 83(4),1125-1130.

Benzie, I.F.F., \& Strain, J.J. (1996). The ferric reducing ability of plasma (FRAP) as a measure of "Antioxidant power": The FRAP assay, Anales Biochemestry, 239(1), 70-76

Budryn, G., Nebesny, E., Podsddek, A., Zyzelewicz, D. \& Materska, M. (2009). Effect of different extraction methods on the recovery of chlorogenic acids, caffeine and Maillard reaction products in coffee beans, European Food Research and Technology, 228, 913-22.
Chávez, K., Medina, L. \& Gámez, N. (2012). Compuestos fenólicos, melanoidinas y actividad antioxidante de café verde y procesado de las especies Coffea arabica y Coffea canephora. Biotecnia, 15 (1), 51-56.

Comisión Venezolana de Normas Industriales. COVENIN. (1995). Norma Venezolana $\mathrm{N}^{\circ}$ 383- 1995. Café Verde en Sacos. Método de Muestreo (Primera Revisión). Fondonorma, Caracas. Venezuela.

Fu, L., Xu, B.T., Xu, X.R., Gan, R.Y., Zhang, Y., Xia, E.Q. \& Li, H.B. (2011). Antioxidant capacities and total phenolic contents of 62 fruits, Food Chemistry, 129(1),345-350.

Gan, R.Y, Li, H.B., Gunaratne, A., Sui, Z.Q. \& Corke, H. (2017). Effects of fermented edible seeds and their products on human health: Bioactive components and bioactivities. Food Scientific. 16:489-531.

Garay, A. (2014). Estandarización de la tostión de café tipo pasilla de máquinas a través de cromatografía líquida de alta eficiencia en la procesadora de café cafetal del río. (Tesis de especialización). Universidad Nacional Abierta, Escuela de Ciencias Básicas, Tecnología e Ingeniería, Ibagué-Colombia.

García, E., Fernández, I. \& Fuentes, A. (2016). Determinación de polifenoles totales por el método de Folin-Ciocalteu, Revista Colombiana de Ciencias Quimico Farmacéuticas, 45 (1), $109-126$.

Gutiérrez, A. (2002). Café, antioxidantes y protección a la salud. Revista Medisan, 6 (4): 72 - 81 Jeszka-Skowron, M., Zgola-Grzeskowiak, A. \& Grzeskowiak, T. (2015). Analytical methods applied for the characterization and the determination of bioactive compounds in coffee, European Food Research and Technology, 24(1), 19-31.

Kocadağlıa, T. \& Gökmen, V. (2016). Effect of roasting and brewing on the antioxidant capacity of espresso brews determined by the QUENCHER procedure, Food Research International, 23(1), 234-256. 
Król, K., Gantner, M., Tatarak, M. \& E, Hallmann. (2020). The content of polyphenols in coffee beans as roasting, origin and storage effect, European Food Research and Technology, 246, 33-39.

Lazcano E., Trejo M., Vargas M. \& Pascual S. (2015). Contenido de fenoles, cafeína y capacidad antioxidante de granos de café verdes y tostados de diferentes estados de México. Revista Ibereroamericana de Tecnología Postcosecha, 16 (2), $293-298$.

Ludwig, I.A, Sánchez, L., Caemmerer, B., Kroh, L.W. \& De Peña, M. (2012). Extraction of coffee antioxidants: Impact of brewing time and method, Food Research International, 48, 57-64.

Marinova, D., Ribarova, F. \& Atanassova, M. (2005). Total phenolics and total flavonoids in bulgarian fruits and vegetables. Journal of the University of Chemical Technology and Metallurgy, 40 (3), 255-260.

Miranda, G., Ventura, J., Suarez, S. \& Fuertes, C. (2007). Actividad citotóxica y antioxidante de los productos de la reacción de maillard de los sistemas modelo de glucosa, glicina y de glucosa L-lisina. Revista de la Sociedad Quimica de Perú, 73 (4), $215-225$.

Odžaković, B., Dzinic, N., Kukrić, Z. \& Grujić, S. (2016). Efecto del grado de tostado sobre la actividad antioxidante de diferentes clases de calidad del café arábica. Acta Scientific Polonorum Technology Aliment, 15 (4), 409-417
Ramírez, M., Plaza, A., Azeredo, M., Balaban, M. \& Marshall, M. (2011). Physicochemical and phytochemical properties of cold and hot water extraction from Hibiscus sabdariffa. Journal Food Scientific, 76(3), 428-435.

Reyes-Luengas, A., Salinas-Moreno, Y., Ovando-Cruz, M. \& Arteaga-Garibay, R. (2015). Analysis of phenolic acids and antioxidant activity of aqueous extracts of jamaica (Hibiscus sabdariffa L.) varieties with calyxes of different colors, Agrociencia, 49, 277-290.

Ribeiro, A., Alvarenga, R., Andrade, S., Silveira, S. \& Borges, F. (2010). Compostos bioativos do café: atividade antioxidante in vitro do café verde e torrado antes e após a descafeinação. Revista Quimica Nova, 33 (1), 20 - 24.

Shang, Y. F., Xu, J. L., Xu, W.L. \& Um, B.M. (2017). Antioxidative polyphenolics obtained from spent coffee grounds by pressurized liquid extraction, South African Journal Botanic, 109, 75-80.

Schenker, S., Heinemann, C., Huber, M., Pompizzi, R., Perren, R. \& Scher, F.E. (2002). Impact of Roasting Conditions on the Formation of Aroma Compounds in Coffee Beans. Journal of Food Science, 67(1), 60-67.

Soler-Rivas, C., Espín, J. \& Wichers, H. (2000). An easy and fast test to compare total free radical scavenger capacity of foodstuffs. Photochemistry Anales, 11(1), 330-338. 